[The activities of amateur societies and groups, including coordination on a national scale, were covered in five contributions. - Eds.]

\title{
Twenty Years of the Japan Amateur Astronomers' Convention
}

Seiji Kimura

Kita Otsuka 2-33-19, Toshima-ku, Tokyo, Japan

In 1967, the Kawasaki Astronomy Club organized the first national assembly for amateur astronomers. This was such a success that a second was held in 1969 and yearly thereafter. Since the establishment in 1982 of the Kaho Prize commemorating Kaho, who was the first Japanese to discover a new comet (in 1936), this award has been presented at the Convention.

\section{Astronomy in the Big Apple}

\section{John Pazmino}

Amateur Astronomers' Association, 1010 Park Avenue,

New York, NY 10028, U.S.A.

New York is probably unique in metropolitan cities worldwide in that it has no "official" or "public" astronomical institution. The Amateur Astronomers' Association, an amateur body, fulfils that role and answers many queries from the general public and various organisations. Founded in 1927, it has around 500 members. Apart from providing all the usual services to its members, it also organizes regular observing sessions in parks and open spaces away from the worst of the light-pollution. It staged very successful public Halley watches in which over 10000 visitors participated.

\section{The Activities of Astronomical Clubs}

\section{Christian Bourdeille}

5 av. Carnot, F-77220 Gretz-Armainvilliers, France

Recent studies have shown that there are some 60000 amateur astronomers in France. This has been reflected in the number of astronomical clubs that have been formed. This contribution summarized the aims and activities of such clubs, the conditions for their success, and their contribution to scientific astronomy. 